\title{
LASER-BASED SLAM WITH EFFICIENT OCCUPANCY LIKELIHOOD MAP LEARNING FOR DYNAMIC INDOOR SCENES
}

\author{
Li Li, Jian Yao*, Renping Xie, Jinge Tu, Chen Feng \\ School of Remote Sensing and Information Engineering, Wuhan University, Wuhan, Hubei, P.R. China \\ Email: jian.yao@whu.edu.cn Web: http://cvrs.whu.edu.cn/
}

\author{
Commission THS, WG ThS9
}

KEY WORDS: Scan Matching, Unmanned Ground Vehicle (UGV), Occupancy Likelihood Map, Simultaneous Localization and Mapping (SLAM)

\begin{abstract}
:
Location-Based Services (LBS) have attracted growing attention in recent years, especially in indoor environments. The fundamental technique of LBS is the map building for unknown environments, this technique also named as simultaneous localization and mapping (SLAM) in robotic society. In this paper, we propose a novel approach for SLAM in dynamic indoor scenes based on a 2D laser scanner mounted on a mobile Unmanned Ground Vehicle (UGV) with the help of the grid-based occupancy likelihood map. Instead of applying scan matching in two adjacent scans, we propose to match current scan with the occupancy likelihood map learned from all previous scans in multiple scales to avoid the accumulation of matching errors. Due to that the acquisition of the points in a scan is sequential but not simultaneous, there unavoidably exists the scan distortion at different extents. To compensate the scan distortion caused by the motion of the UGV, we propose to integrate a velocity of a laser range finder (LRF) into the scan matching optimization framework. Besides, to reduce the effect of dynamic objects such as walking pedestrians often existed in indoor scenes as much as possible, we propose a new occupancy likelihood map learning strategy by increasing or decreasing the probability of each occupancy grid after each scan matching. Experimental results in several challenged indoor scenes demonstrate that our proposed approach is capable of providing high-precision SLAM results.
\end{abstract}

\section{INTRODUCTION}

In outdoor environments, the Global Navigation Satellite System (GNSS) can provide accuracy position and navigation services for the robots. However, in indoor environments, GNSS is unavailable due to the signals are very weak or even not received, which results in that the moving location of the robots in an indoor scene cannot be simply obtained. For an intelligent robot, it must know itself where it is before it makes the next decision and completes a specific task. In general, this problem is formulated as the simultaneous localization and mapping (SLAM) in the robotics society, which is a chicken and egg problem. It is a process of building and updating a map of an unknown environment based on the data collected by the sensors mounted on some mobile robot platform (e.g., a mobile Unmanned Ground Vehicle (UGV) used in this paper), and simultaneously determining itself where it is located on the map.

SLAM is a classical and fundamental problem in the fields of robotics society and computer vision, and has received increasing attention over recent years. In efforts to solve this problem, a variety of methods based on different sensors have been developed. Those approaches can be divided into three main categories: vision-based [16], RGBD-based and LiDAR-based approaches. Vision-based SLAM is the cheapest approach, which only uses a monocular camera or stereo one to achieve autonomous navigation and mapping in unknown environments $[16,11,6]$, but it may be failed in the regions with poor texture. To improve the robustness of SLAM in poorly-textured regions, many researches had developed a series of robust techniques based on RGBD cameras, such as Kinect sensor [10, 8]. However, these RGBDbased approaches are only suitable for relatively small indoor scenes due to the effective range of most popular RGBD cameras.

${ }^{*}$ Corresponding author
Another kind of representative approaches is the use of a laser scanner [15, 27, 26], which has been successfully used on UGVs, Unmanned Surface Vehicles (USVs) and Micro Air Vehicles (MAVs), and so on. Our work in this paper also applies the laser scan data collected by a laser scanner mounted on a UGV to solve the SLAM problem for the indoor environments.

Laser scan matching is one of the popularly used methods and has been proved to be effective for localizing the robot's position. In general, working on localization using laser scan matching starts with the Iterative Closet Point (ICP) algorithm [3], which iteratively optimizes the rigid-transformation matrix by minimizing the sum of distances between two clouds of points. In many cases, the ICP-based approaches can obtain matching results with a high accuracy. However, applying the ICP algorithm for scan matching is time consuming due to that it needs to search corresponding points for each point in each iteration. To avoid searching for point associations, Diosi and Kleeman [9] proposed a novel method for 2D laser scan matching called Polar Scan Matching (PSM) which takes advantage of the natural polar coordinate system of the laser scans to finish the scan matching. However, the point-to-point matching approaches may be failed in some cases due to the sum of distances between points is not a good metric for evaluating the difference of two scans. For example, in some local regions, the densities of two scans are different due to that the distances of those points to the center of the laser scanner vary dramatically, so maybe we can't find the ideal corresponding points between two scans based on the closest distance principle. Censi [7] proposed the Iterative Closed Line (ICL) algorithm, which is an ICP variant and minimizes the point-to-line distance instead of the point-to-point one used in the basic ICP. In addition, Biber and Straßer [5] developed a new approach for 2D laser scan matching based on Normal Distributions Transform (NDT) which is a new representation of a set of points. Olson [19] proposed a 


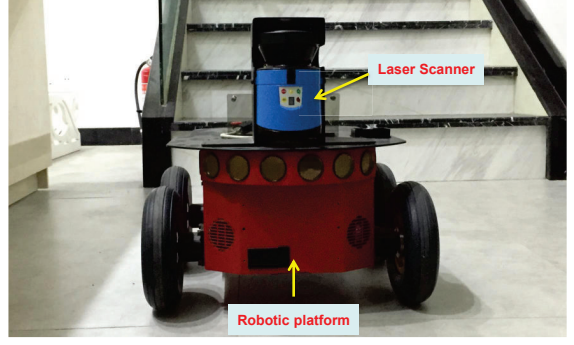

Figure 1: A photo of the mobile UGV used in our paper, which is comprised of a laser scanner SICK LMS-100 and a robotic platform Pioneer 3-AT.

real-time correlative scan matching approach by formulating this problem in a probabilistic framework, which used an exhaustive sampling based approach to find the rigid-body transformation that maximizes the probability of the observed data.

However, most of scan matching methods above mentioned generally operate on consecutive pairs of scans, without maintaining any historical data. Therefore, any small error in each scan matching will be retained and accumulated as time goes on, resulting in drift. To solve this issue, many people proposed to apply the grid-map based method to process the laser scan matching [12, 20, 1, 22, 15, 2, 23, 24, 18, 21]. Generally, it first generates a grid-based occupancy likelihood map based on all previous scans, and then applies the scan-to-map matching to find the optimal rigid-body transformation which is used to align the points of current scan to the whole map. Kohlbrecher et al. [15] developed a system for fast learning of occupancy grid maps by using a fast approximation of map gradients and a multi-resolution grid. They applied the Gauss-Newton approach to find the rigid-body transformation between current scan with the existing map learned from all previous scans. Bachrach et al. [2] proposed a novel estimation, navigation and control system for a MAV system in GPS-denied environments. It follows the contour-slope model proposed in [20] of measurement likelihood of subsequent scans as a generative probabilistic model given by a Gaussian blur of a polygonal reduction of previous scans, which was also utilized in [1]. Tang et al. [23] developed a mobile UGV indoor position system using the gridbased laser scan matching with an improved probabilisticallymotivated Maximum Likelihood Estimation (IMLE) algorithm. Their developed system applies a totally brute search matching method to find the rigid-body transformation between current scan and the likelihood grid map with refined search scope which is provided by the ICP algorithm. They also proposed a simple line-feature-based and three-level strategy of likelihood value determination to generate the likelihood map by combining the grid-point occupation method [22] and the contour-slope method [20]. However, we find that the above methods generate the likelihood map by only applying 1-D (1-Dimension) Gaussian blur along the contours or the points and then find the optimal transformation between current laser scan and the existing map via the brute search method or the gradient ascent method. These above methods haven't considered the effect of dynamic objects existed in the indoor environments, so may be failed in the dynamic scenes.

In addition, most of SLAM methods assume that all the points in one scan are acquired simultaneously while most of the available rangefinders measure points by rotation sequentially. Hence, those methods may be failed under a fast motion due to that they ignore the scan distortion caused by the motion of robots. Bezet and Cherfaoui [4] proposed a method to correct the error produced by time stamping error in sensor data acquisition, and obtain the data at the same time. Hong et al. [14] proposed a novel scan matching method called VICP which is a family of ICP algorithms. In this algorithm, they estimated and updated a velocity of a rangefinder numerically over ICP iterations. In this way, the distortion of a scan which comes from time differences during scanning is compensated by using the estimated velocity refined in each iteration. Zhang and Singh [26] modeled the LiDAR motion with constant angular and linear velocities during a sweep. They simply linearly interpolated the pose transform within a sweep for the points received at different times, and then solved the LiDAR motion with the Levenberg-Marquardt method [13].

In this paper, we propose a novel approach for SLAM in dynamic indoor scenes based on a 2D laser scanner mounted on a mobile UGV aided by the grid-based occupancy likelihood map. Firstly, we propose a new method to generate the occupancy likelihood map via applying a 2-D Gaussian blurring along the contours, which is more reasonable than only using a 1-D Gaussian blurring. Secondly, to compensate the scan distortion caused by the motion of the robot platform, we propose to integrate a velocity of a laser range finder (LRF) into the scan matching optimization framework. Thirdly, to ensure the accuracy and robustness of the recovered transformation, we propose to combine the brute search and gradient ascent to find the optimal solution. At last, to reduce the influence of dynamic objects existed in indoor environments as much as possible, we propose a new occupancy likelihood map learning strategy inspired by the background modeling algorithm [25]. The probability value of each grid will be increased or decreased after each scan matching. We tested our proposed SLAM approach in several challenged indoor environments, whose experimental results sufficiently demonstrate that our approach can generate the whole map without drift in most cases and localize the robot's positions everywhere with a high accuracy.

\section{OUR APPROACH}

\subsection{Algorithm Overview}

Given two sequential laser range scans $S_{t-1}$ and $S_{t}$ at times $t-1$ and $t$, the laser scan matching algorithm is used to find the rigidbody transformation $\mathrm{T}$ that aligns the current laser scan with the previous one. However, if we apply this method for each pair of laser scans one by one, the pose drifting problem will appear due to that the matching error between two laser scans will be accumulated gradually and affect the accuracy of next matching.

In our work, rather than explicitly matching pairs of scans $S_{t-1}$ and $S_{t}$, we match current scan $S_{t}$ to a grid-based occupancy likelihood map $\mathbb{M}_{t-1}$ generated by all previous scans. We use probabilistic scan-matching algorithms due to their robustness to large discontinuities in the range measurements. Each cell of the map $\mathbb{M}_{t-1}$ stores the likelihood value of the space region in $2 \mathrm{D}$ space measured by the laser points in all previous scans. Assuming that each point measurement in a laser scan is independent, according to Bayes's rules, the sum likelihood of an entire scan can be computed as:

$$
P\left(S_{t} \mid \mathbb{M}_{t-1}\right)=\sum_{\mathbf{x} \in S_{t}} P\left(\mathbf{x} \mid \mathbb{M}_{t-1}\right)
$$

where $P\left(\mathbf{x} \mid \mathbb{M}_{t-1}\right)$ represents the probability of the point $\mathbf{x} \in S_{t}$ at that location in the map $\mathbb{M}_{t-1}$. The best rigid-body transformation $\mathrm{T}^{*}$ can be found from all candidates by maximizing the likelihood of the laser scan according to:

$$
\left.\mathrm{T}^{*}=\underset{\mathrm{T}}{\operatorname{argmax}}\left(P\left(\mathrm{~T} \otimes S_{t} \mid \mathbb{M}_{t-1}\right)\right)\right)
$$




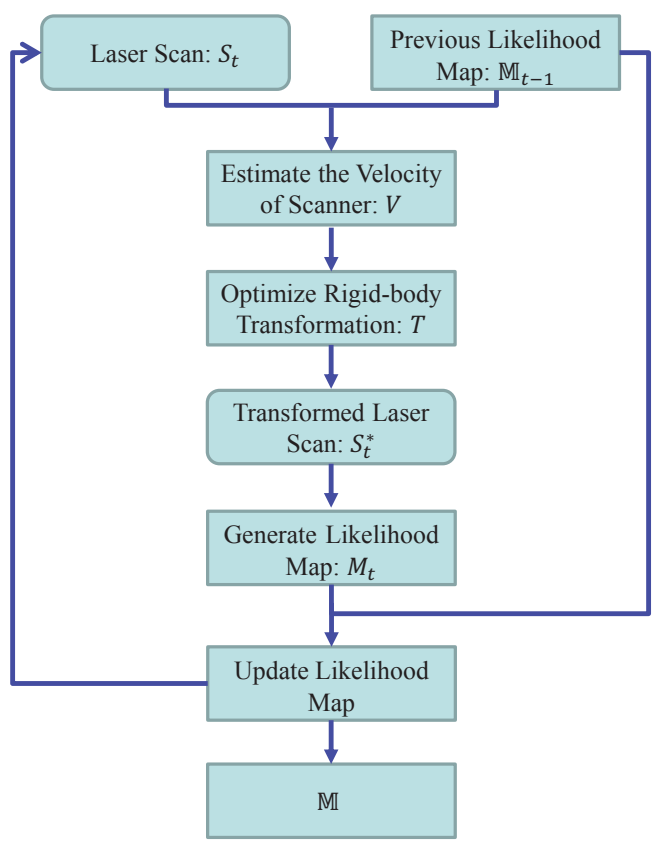

Figure 2: The flowchart of our proposed SLAM algorithm.

where $\mathrm{T} \otimes S_{t}$ is the set of laser points $S_{t}$ transformed by the rigid body transformation $\mathrm{T}$.

The key issues of a grid-based probabilistic scan-matching algorithm can be summarized as follow: the generation of the occupancy likelihood map from all previous maps, and an optimization framework that allows us to find the optimal rigidbody transformation. In this paper, we propose a series of efficient techniques to solve those problems. Figure 1 shows the mobile UGV with a single laser scanner SICK LMS-100 used in this paper. The flowchart of our proposed SLAM algorithm is illustrated in Figure 2. Let $S_{t}$ be the current laser scan at the time $t$ and $\mathbb{M}_{t-1}$ be the occupancy likelihood map at the previous time $t-1$, which is learned from all previous scans. Firstly, we estimate the rigid-body transformation $\mathrm{T}$ between the current scan $S_{t}$ with the previous map $\mathbb{M}_{t-1}$ by combining the brute search and the gradient ascent implemented by the Levenberg-Marquardt (LM) algorithm. At the same time, to reduce the influence of the scan distortion caused by the motion of the UGV, we integrate the velocity of the laser scanner into the LM optimization framework. Secondly, we transform $S_{t}$ into the same coordinate system of $\mathbb{M}_{t-1}$ based on the rigidbody transformation $\mathrm{T}$, which is denoted as $S_{t}^{*}=\mathrm{T} \otimes S_{t}$. The likelihood map $\mathrm{M}_{t}$ of $S_{t}^{*}$ can be generated by applying the 2-dimension Gaussian blurring operation along the contours connected by the points of the laser scan $S_{t}^{*}$. At last, we learn the new occupancy likelihood map $\mathbb{M}_{t}$ at the current time $t$ via a novel strategy inspired by the background subtraction method proposed by Yao and Odobez [25]. All the above steps are repeated with the acquisition of laser scans and the final occupancy likelihood map $\mathbb{M}$ can be robustly achieved.

\subsection{Likelihood Map Generation}

The fundamental component of the grid-based scan matching algorithm is the occupancy likelihood map generated from all previous scans, which allows us to compute the occupancy likelihood of each point. One common approach for map generation is to first store all points from all previous scans. Then, for each point $\mathrm{x}$ in the current scan $S_{t}$, the distance to the closest point $\mathbf{x}^{\prime}$ is computed. Next, we apply the Gaussian probability model to simulate the laser measurement noise. In this way, the occupancy likelihood of each point can be defined as:

$$
P\left(\mathbf{x} \mid \mathbb{M}_{t-1}\right) \propto \exp \left(-d\left(\mathbf{x}, \mathbf{x}^{\prime}\right) / \sigma\right),
$$

where $d\left(\mathbf{x}, \mathbf{x}^{\prime}\right)$ denotes the distance between $\mathbf{x}$ and $\mathbf{x}^{\prime}$, and $\sigma$ stands for the standard deviation of the sensor measurement noise.

However, this approach probably fails in some situations due to that it attempts to find correspondences for all points in the scans even though a number of points may not correspond to the current scan. As we know, the main structure of indoor environments is comprised of planar surfaces, which results in a set of piecewise linear line segments in a 2D laser scan. For the points in the current scan, we maybe can't find the corresponding points in all previous scans, but have the bigger chance to locate on the same surfaces represented by contours. Therefore, for a point $\mathbf{x}$ in the current scan, computing its occupancy likelihood with the distance $d(\mathbf{x}, \mathbb{C})$ to the closest contour $\mathbb{C}$ extracted from all previous scans is more reasonable, such as

$$
P\left(\mathbf{x} \mid \mathbb{M}_{t-1}\right) \propto \exp (-d(\mathbf{x}, \mathbb{C}) / \sigma) .
$$

In this contour-based approach, the grids crossed by the contours have the same and the biggest probability, and the occupancy likelihood values of the remaining grids are obtained by applying 1-D Gaussian blurring along the contours. An example of the last likelihood map is illustrated in Figure 3(b). However, this approach assume that the grids crossed by the contours are all the robust features in this environment, and completely ignores the error of the contours. Sometimes, the error of contour connection maybe occurs, especially when the distance between two adjacent points become longer with increasing of the point distance to the center of the laser scanner. For example, we assume that there is an open door on the wall, and two adjacent wall points located in different sides of an open door are possibly connected into a same contour which represents the wall. In this way, the grids covered by this door region are regarded as robust features. Obviously, it is unreasonable. Thus, in some cases, the traditional contour-slope model maybe can't accurately represent the scanned scenes.

In this paper, we propose a new improved contour-based approach and combine the above-mentioned two common map generation approaches. Firstly, all contours from the laser scan need to be extracted. All distances $\left\{d_{k} \mid d_{k}=\left\|\mathbf{x}_{k}-\mathbf{x}_{k-1}\right\|\right\}_{k=2}^{K}$ between all pairs of adjacent points are sorted in an ascending order, where $K$ denotes the number of all points in the laser scan. A pair of adjacent points $\left(\mathbf{x}_{k-1}, \mathbf{x}_{k}\right)$ is selected as the seed of an initial contour if it satisfies following constraints: $d_{k}$ is the smallest and smaller than the predefined distance threshold, and $\mathbf{x}_{k-1}$ or $\mathbf{x}_{k}$ is not jointed by other extracted contours. The adjacent points $\left\{\mathbf{x}_{i}\right\}$ are iteratively joined until no more ones satisfy the following constraints:

$$
\left\{\begin{aligned}
d_{i} & \leq d_{t h}, \\
\frac{\max \left(d_{i}, d_{j}\right)}{\min \left(d_{i}, d_{j}\right)} & \leq r_{t h},
\end{aligned}\right.
$$

where $d_{i}$ denotes the distance between $\mathbf{x}_{i-1}$ and $\mathbf{x}_{i}$, i.e., $d_{i}=$ $\left\|\mathbf{x}_{i}-\mathbf{x}_{i-1}\right\|, \mathbf{x}_{j}$ stands for one of the endpoints of the current contour, $\mathbf{x}_{i}$ is next to $\mathbf{x}_{j}$, and $d_{t h}$ and $r_{t h}$ are two thresholds for the distance and the distance ratio, respectively, which were set as $d_{t h}=150 \mathrm{~mm}$ and $r_{t h}=2$ in this paper. After collecting one contour, we iteratively repeat the above procedures to find new contours from the remaining points of the scan. 


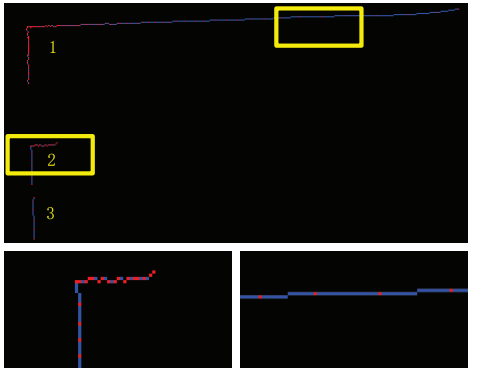

(a)

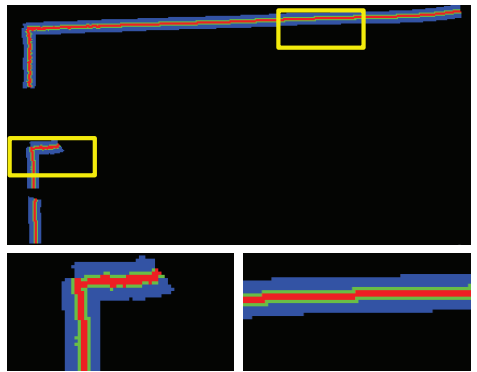

(b)

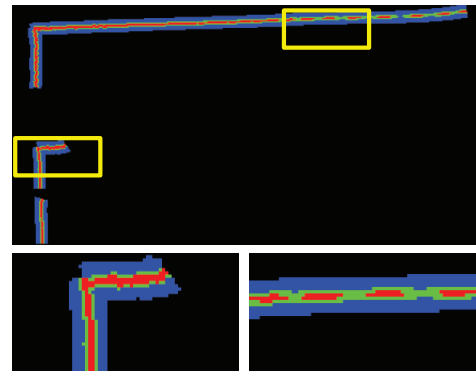

(c)

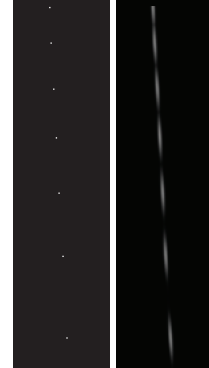

(d)

Figure 3: Illustrations of the contour extraction and the occupancy likelihood map generation: (a) three contours in blue extracted from the raw laser measurements alongside the raw laser readings in red dots; (b) the likelihood map generated from the contours by applying a 1-Dimension Gaussian blurring operation along the contour using the method presented in [20]; (c) the occupancy likelihood map generated by our proposed method; (d) a detailed view for our proposed method (Left: the original laser points in a contour; Right: the occupancy likelihood map by applying our propsed 2D Gaussian blurring operation).
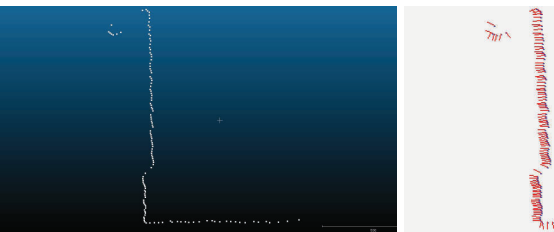

Figure 4: An illustration of the normal estimation by RDPCA:(Left) the partial points of a laser scan; (Right) the corresponding orientations of the point normals marked in red lines, which were computed by RDPCA.

Compared with the traditional contour-based approach, we replace the 1-D Gaussian blurring operation with the 2-D one. For each pair of points $\left(\mathbf{x}_{i}, \mathbf{x}_{j}\right)$ in the same contour, we regard the direction of the line connecting $\mathbf{x}_{i}$ and $\mathbf{x}_{j}$ as the $x$-axis and the $y$-axis is defined as the vertical direction of the $x$-axis. The likelihood of a point $\mathrm{x}$ is computed as follow:

$$
P(\mathbf{x} \mid \mathbb{M}) \propto \exp \left(-d_{x}(\mathbf{x}, \mathbb{C}) / \sigma_{x}-d_{y}(\mathbf{x}, \mathbb{C}) / \sigma_{y}\right),
$$

where $\sigma_{x}$ and $\sigma_{y}$ stand for two standard deviations in the $x$ - and $y$-axes, respectively, which were set as 1.5 and 10 in this paper. $d_{x}(\mathbf{x}, \mathbb{C})$ and $d_{y}(\mathbf{x}, \mathbb{C})$ denote the distances to the contour along the $x$ - and $y$-axes, respectively, which are computed as follow:

$$
\left\{\begin{array}{l}
d_{x}(\mathbf{x}, \mathbb{C})=\min _{\mathbf{x}_{k} \in \mathbb{C}}\left(d_{x}\left(\mathbf{x}, \mathbf{x}_{k}\right)\right), \\
d_{y}(\mathbf{x}, \mathbb{C})=d(\mathbf{x}, \mathbb{C}),
\end{array}\right.
$$

where $d_{x}\left(\mathbf{x}, \mathbf{x}_{k}\right)$ represents the distance from $\mathbf{x}$ to $\mathbf{x}_{k}$ along the direction of the $x$-axis. Figure 3(c) shows an example of the occupancy likelihood map created by this new approach. From Figure 3(a), we can find that this laser scan is comprised of three contours, which are numbered as 1, 2 and 3, respectively. The distance between two adjacent points becomes bigger as the extension of contours, especially for the longest contour numbered as 1. Compared with the occupancy likelihood map shown in Figure 3(b), we can find that the occupancy likelihood value of each grid crossed by the contour is not always the same and the biggest as shown in Figure 3(c), which is also determined by the distance to the closest point along the $x$-axis, especially in the local regions marked by the upper-right box. But if the distance between two points is small, the likelihood map is similar for two methods, as shown in the local region marked by the lower-left box. Our proposed 2D Gaussian blurring results are more easily observed in a detailed view of partial contour as shown in Figure 3(d).

\subsection{Scan-to-Map Matching}

Scan matching is the process of aligning laser scans with each other or with an existing occupancy likelihood map. Our approach aligns the new laser scan to the likelihood map learned from all previous scans as described in Section 2.4. Given the previous occupancy likelihood map $\mathbb{M}_{t-1}$ and the current laser scan $S_{t}$, the scan-to-map matching aims to find the optimal rigidbody transformation $\mathrm{T}^{*}$ between $S_{t}$ and $\mathbb{M}_{t-1}$ by minimizing the following energy function:

$$
E\left(S_{t}, \mathbb{M}_{t-1}\right)=\sum_{\mathbf{x} \in S_{t}} w(\mathbf{x})\left(1-P\left(\mathrm{~T} \otimes \mathbf{x} \mid \mathbb{M}_{t-1}\right)\right)^{2},
$$

where $\mathrm{x}$ stands for one point in the scan $S_{t}$, the operation $\mathrm{T} \otimes \mathrm{x}$ means that the point $\mathbf{x}$ is transformed by $\mathrm{T}$, and $P\left(\mathrm{~T} \otimes \mathbf{x} \mid \mathbb{M}_{t-1}\right)$ denotes the occupancy likelihood value of the transformed position in $\mathbb{M}_{t-1}$. We know that the robustness of the recovered rigidbody transformation depends on at least two non-planar surfaces. To efficiently balance the contribution of surfaces with different orientations, we propose to set different weights for different points. The weight $w(\mathbf{x})$ of the point $\mathbf{x}$ is used to balance the influence of each point in the optimization framework, which is computed as follows. We first compute the normal $\mathbf{n}=$ $\left(n_{x}, n_{y}\right)^{\top}$ of each point $\mathbf{x}$ via the Robust Diagnostic PCA (RDPCA) algorithm [17], as shown in Figure 4, and then compute the histogram of normal orientations $\mathcal{H}$ comprised of $B$ bins where $B=12$ used in this paper. In indoor scenes, there often exists one or several main planar surfaces in a laser scan due to the limited viewpoint and measurement distance. The points in each bin have the same weight $w_{b}$ defined by the following Sigmoid function:

$$
w_{b}=\frac{1}{\mathcal{H}_{b}} \times \frac{1}{1+\exp \left(\left(100-\mathcal{H}_{b}\right) / 50\right)}
$$

where $\mathcal{H}_{b}$ stands for the frequency of the $b$-the bin in $\mathcal{H}$.

Generally, there are mainly two ways to solve the above optimization problem: the brute search and gradient ascent methods. The brute search method searches the best transformation from all candidate transformations, but this method is inefficient when the search windows are large and the search steps are small. The gradient ascent method can find the optimal transformation in most cases more efficiently, but it has inherent risk of getting stuck in local minimum. So, it always needs a relatively accurate initial transformation to reduce this risk. In this paper, to find the optimal transformation more accurately and efficiently, we combine the brute search method and the LM algorithm which is the gradient ascent method in nature to find the optimal transformation. Before applying the LM optimization algorithm, 
we find the initial rough estimated transformation via the brute search method with narrow search windows $\left(w_{\theta}, w_{x}, w_{y}\right)$, and large search steps $\left(s_{\theta}, s_{x}, s_{y}\right)$ in the three parameters of a rigidbody transformation, i.e., a rotation angle $\theta$, two translations $t_{x}$ and $t_{y}$ in the $x$ - and $y$-axes.

However, in the above mentioned scan-to-map matching approach, the laser scan distortion caused by the motion of the UGV has been ignored, so this approach is prone to be erroneous under a fast motion. This problem can be solved by compensating the scan distortion of the laser scan as VICP [14] does. In the VICP algorithm, it first estimates the velocity of the rangefinder and corrects the scan distortion at the same time, and then applies the ICP algorithm to finish the process of scan matching. In our approach, we integrate the estimated velocity into the LM optimization framework instead of the ICP algorithm.

In order to compensate the scan distortion, the velocity of the rangefinger has to be estimated. Let $\mathrm{T}_{t}$ and $\mathrm{T}_{t-1}$ be the rigidbody transformation of the laser scan $S_{t}$ and $S_{t-1}$ with respective to the map $\mathbb{M}$. So the relation between $S_{t}$ and $S_{t-1}$ can be represented as:

$$
\mathbf{x}_{k}^{t-1}=\mathrm{T}_{t-1}^{-1} \mathrm{~T}_{t} \mathbf{x}_{k}^{t}, k=1,2, \cdots, K,
$$

where $\mathbf{x}_{k}^{t-1}$ and $\mathbf{x}_{k}^{t}$ represent the points in the laser scans $S_{t-1}$ and $S_{t}$, respectively, and $K$ is the number of points in one laser scan. We assume that the velocity is constant during the scan time $\Delta t$. According to the method presented in VICP, we approximate $V_{t}$, i.e., the velocity of the rangefiner at the time $t$, as:

$$
V_{t}=\frac{1}{\Delta t} \log \mathrm{T}_{t-1}^{-1} \mathrm{~T}_{t} .
$$

Then we use the estimated velocity to compensate the points in $S_{t}$ one by one as follows:

$$
\hat{\mathbf{x}}_{k}^{t}=e^{k \Delta t_{s} V_{t}} \mathbf{x}_{k}^{t},
$$

where $\hat{\mathbf{x}}_{k}^{t}$ stands for the point after compensation and $\Delta t_{s}=\frac{\Delta t}{n}$ representing the elapsed time between two adjacent points.

According to Eq. (12), we can transform all points in $S_{t}$ into the time when the first point of $S_{t}$ is scanned. The compensated points are used to optimize the energy function presented in Eq. (8) in the LM optimization framework. After each iteration of the LM algorithm, we re-estimate the velocity $V_{t}$ and re-calculate the compensated points $\left\{\hat{\mathbf{x}}_{k}^{t}\right\}_{k=1}^{K}$ before the next iteration beginning based on the current transformation $\mathrm{T}_{t}$. In this way, both $V_{t}$ and $\mathrm{T}_{t}$ are iteratively refined simultaneously by using the previous estimated results as the initial estimation for the next iteration in the LM optimization framework. The procedures of our proposed scan matching and compensation algorithm is summarized in Algorithm 1, where $\hat{S}_{t}$ denotes the compensated scan, $I_{\max }$ is the maximal iteration number of the LM algorithm, and $\operatorname{LM}\left(\hat{S}_{t}, \mathbb{M}_{t-1}, \mathrm{~T}_{t}\right)$ denotes one optimization iteration in the LM algorithm.

\subsection{Likelihood Map Learning}

Given the current laser scan $S_{t}$ and the transformation $\mathrm{T}_{t}$ obtained by the scan-to-map matching algorithm, the occupancy likelihood map $\mathbb{M}_{t-1}$ need to be updated before the use of aligning the next laser scan, and the newly updated map is defined as $\mathbb{M}_{t}$. Generally, we need to regenerate the occupancy likelihood map based on the stored points or contours. However, this method needs to operate the process of the likelihood map generation before each laser scan matching and also needs large memory to store the points or contours and high computation. In addition, the

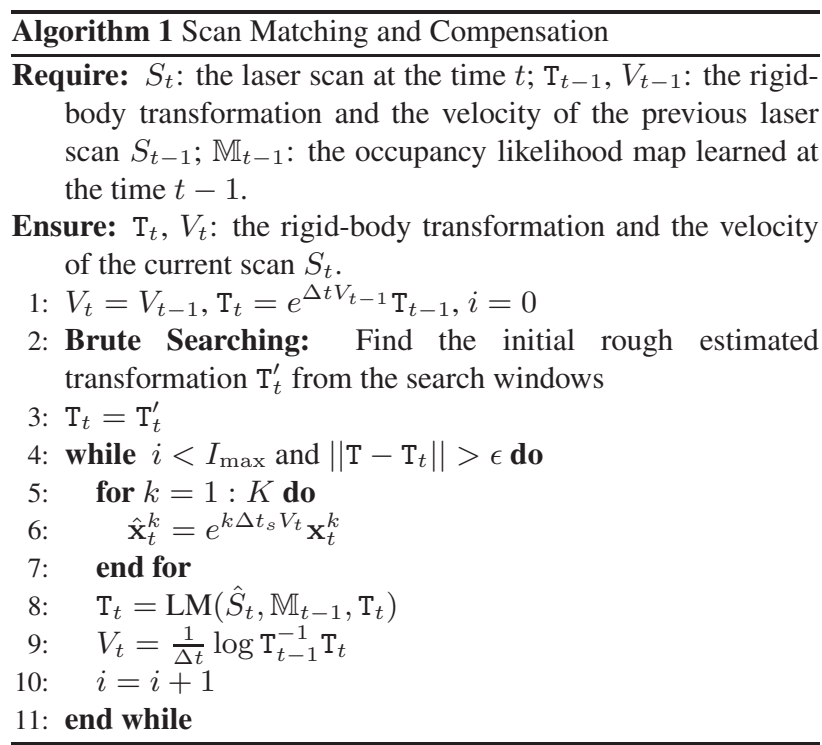

biggest disadvantage of this method is that it hasn't considered the influence of dynamic objects in indoor environments. In this paper, we propose a new strategy inspired by the background subtraction method [25] to update the occupancy likelihood map. Background subtraction is an effective technique to detect the foreground objects from a video stream captured from a stationary camera. The basic component of the background subtraction method is the background model learned from all previous image frames. The background model needs to be updated while new image frame comes. The similar strategy used in background subtraction can be applied in the updating and learning process of the occupancy likelihood map.

While at the first scanning time $t=1$, the occupancy likelihood map $\mathbb{M}_{t}$ is directly set as the likelihood map of $S_{t}$, i.e., $\mathbb{M}_{t}=\mathbb{M}_{t}$. In the sequential laser scans, the new occupancy likelihood map is updated as follows. We first align the laser scan $S_{t}$ into the coordinate system of $\mathbb{M}_{t-1}$, and then generate the likelihood map $\mathrm{M}_{t}$ of $S_{t}$ via the approach presented in Section 2.2. For some grid, we need to store three components, i.e., $\mathbb{M}(\mathrm{g})=$ $\left\{\mathbb{M}^{p}(\mathbf{g}), \mathbb{M}^{\hat{p}}(\mathbf{g}), \mathbb{M}^{n}(\mathbf{g})\right\}$, where $\mathbb{M}^{p}(\mathbf{g}) \in[0,1]$ and $\mathbb{M}^{\hat{p}}(\mathbf{g}) \in$ $[0,1]$ stand for the current occupancy likelihood value and the maximal one that the grid $\mathbf{g}$ is achieved in the past, respectively, and $\mathbb{M}^{n}(\mathbf{g})$ denotes the times of this grid that has been observed in all previous laser scans. The likelihood value of each grid will be updated with one of the following four ways:

- No updating: If both the likelihood values $\mathbb{M}_{t-1}^{p}(\mathrm{~g})$ and $\mathbb{M}_{t}^{p}(\mathbf{g})$ in maps $\mathbb{M}_{t-1}$ and $\mathbb{M}_{t}$ are zero, i.e., $\mathbb{M}_{t-1}^{p}(\mathbf{g})=0$ and $\mathrm{M}_{t}^{p}(\mathbf{g})=0$, no updating occurs at this grid $\mathbf{g}$ due to that it is pointless.

- Increasing likelihood: If there exists at least one point located in $\mathbf{g}$ in $\mathrm{M}$, i.e., $\mathrm{M}_{t}^{p}(\mathbf{g})>0$ and $\mathrm{M}^{n}(\mathbf{g}) \geq 1$, we update $\mathbb{M}_{t}(\mathbf{g})$ as follows:

$$
\left\{\begin{array}{l}
\mathbb{M}_{t}^{p}(\mathbf{g})=\left(1-w_{u}\right) \cdot \max \left(\mathbb{M}_{t-1}^{p}(\mathbf{g}), \mathbb{M}_{t}^{p}(\mathbf{g})\right)+w_{u} \\
\mathbb{M}_{t}^{\hat{p}}(\mathbf{g})=\max \left(\mathbb{M}_{t-1}^{\hat{p}}(\mathbf{g}), \mathbb{M}_{t}^{p}(\mathbf{g})\right), \\
\mathbb{M}_{t}^{n}(\mathbf{g})=\mathbb{M}_{t-1}^{n}(\mathbf{g})+1
\end{array}\right.
$$

where $w_{u}$ is the likelihood increasing rate calculated as:

$$
w_{u}=w_{l}\left(1+\tau \mathbb{M}_{t-1}^{\hat{p}}(\mathbf{g})\right),
$$

where $w_{l}$ is the predefined learning rate and $\tau$ is a control coefficient ( $w_{l}=0.01$ and $\tau=3$ used in this paper). 
- Decreasing likelihood: If there exists no point in $\mathrm{g}$ in $\mathrm{M}_{t}$, i.e., $\mathbb{M}_{t}^{p}(\mathbf{g})=0$, we decrease the likelihood value $\mathbb{M}_{t}^{p}(\mathbf{g})$ just while there exist no enough points in $\mathbf{g}$ in previous scans, i.e., $\mathbb{M}_{t-1}^{n} \leq n_{t h}$, as follows:

$$
\mathbb{M}_{t}^{p}(\mathbf{g})=\left(1-w_{d}\right) \mathbb{M}_{t-1}^{p}(\mathbf{g}),
$$

where $w_{d}$ is the decreasing rate defined as follow:

$$
w_{d}=\frac{w_{l}}{1+\tau \mathbb{M}_{t-1}^{\hat{p}}(\mathbf{g})} .
$$

- Likelihood interpolation: The remaining grids are updated by interpolating the values from $\mathbb{G}_{u}$ and $\mathbb{G}_{d}$ standing for the grids increased and decreased, respectively. Given a grid $\mathbf{g}$ to be updated, we compute the increased and the decreased likelihood values $p_{u}$ and $p_{d}$ as follows:

$$
\left\{\begin{array}{l}
p_{u}=\underset{\mathbf{g}_{k} \in \mathbb{G}_{u}}{\operatorname{argmax}}\left(\mathbb{M}_{t}^{p}\left(\mathbf{g}_{k}\right)-\mathbb{M}_{t-1}^{p}(\mathbf{g})\right) \exp \left(\frac{-d\left(\mathbf{g}, \mathbf{g}_{k}\right)}{\sigma_{l}}\right), \\
p_{d}=\underset{\mathbf{g}_{k} \in \mathbb{G}_{d}}{\operatorname{argmax}}\left(\mathbb{M}_{t}^{p}\left(\mathbf{g}_{k}\right)-\mathbb{M}_{t-1}^{p}(\mathbf{g})\right) \exp \left(\frac{-d\left(\mathbf{g}, \mathbf{g}_{k}\right)}{\sigma_{l}}\right),
\end{array}\right.
$$

where $d\left(\mathbf{g}, \mathbf{g}_{k}\right)$ denotes the distance between two grids $\mathbf{g}$ and $\mathbf{g}_{k}$, and $\sigma_{l}$ denotes the standard deviation of the used Gaussian interpolation function, which was set as $\sigma_{l}=1.5$ in this paper. We set $p=p_{u}$ if $\left|p_{u}\right|>\left|p_{d}\right|$, otherwise, $p=p_{d}$. Then, $\mathbb{M}_{t}(\mathbf{g})$ is updated as follows:

$$
\left\{\begin{array}{l}
\mathbb{M}_{t}^{p}(\mathbf{g})=\mathbb{M}_{t-1}^{p}(\mathbf{g})+p \\
\mathbb{M}_{t}^{\hat{p}}(\mathbf{g})=\max \left(\mathbb{M}_{t-1}^{\hat{p}}(\mathbf{g}), \mathbb{M}_{t}^{p}(\mathbf{g})\right) \\
\mathbb{M}_{t}^{n}(\mathbf{g})=\mathbb{M}_{t-1}^{n}(\mathbf{g})+1
\end{array}\right.
$$

According to the above updating strategy, if one grid in $\mathbb{M}_{t-1}$ is occupied by the points of a dynamic object, the likelihood of this gird will be decreased gradually during map updating. In contrast, the likelihood will be increased. In this way, the likelihood values of dynamic objects become smaller but those of static objects become bigger in the last map $\mathbb{M}$.

\subsection{Multi-Resolution Map Representation}

Until now, we have presented a complete grid-based probabilistic scan-matching algorithm in a certain resolution. Although we find a rough estimated transformation for the LM optimization algorithm via brute searching. But it also has risk of getting stuck in local minimum. To alleviate this problem as far as possible, we also apply a multi-resolution map representation similar to the pyramid approaches used in computer vision. The resolution of each fine grid-map is double of the preceding coarse one. Our proposed scan-to-map matching algorithm starts at the coarsest map level whose resulting estimated rigid-body transformation and velocity are used as the initial estimations for the next level until the bottom one. To update the likelihood map for each level via the strategy presented in Section 2.4, we must first know the accurate rigid-body transformation in each level and compute them by transferring the transformation estimated in the bottom level to other based on their resolutions. The updated likelihood maps in all levels are used as the reference maps for the next incoming scan.

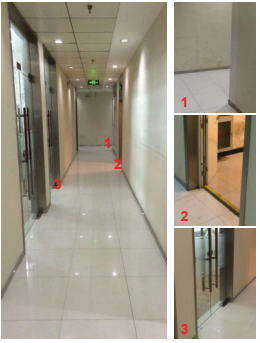

(a)

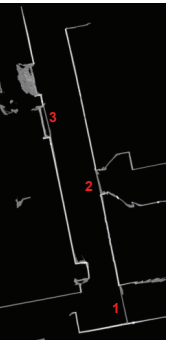

(b)
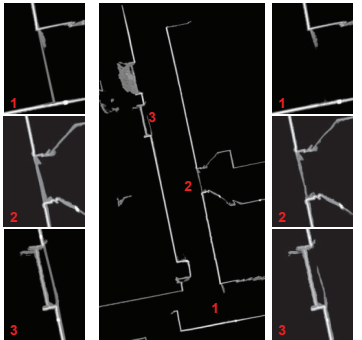

(c)
Figure 5: Comparative results of the traditional and our proposed contour-based likelihood generation approaches: (a) an indoor corridor scene with several sunk doors; (b) the last likelihood map generated by the traditional contour-slope model; (c) the last likelihood map generated by our proposed method. Brighter intensities indicate higher likelihood values.

\section{EXPERIMENTAL RESULTS}

To illustrate that our proposed algorithm is effective, we conducted the experiments in several indoor environments. The experimental data were all collected by the UGV platform presented in Figure 1, whose moving velocity is about $1.2 \mathrm{~m} / \mathrm{s}$. The used laser scanner is SICK LMS-100 with a scanning range $[0.5 \mathrm{~m}, 20 \mathrm{~m}]$, a scanning angle of $270^{\circ}$, a resolution of $0.5^{\circ}$, and an acquisition frequency of $50 \mathrm{~Hz}$. In total, there are 540 points in each laser scan.

Figure 5 shows the comparative results of the occupancy likelihood maps generated by the traditional contour-slope model and our proposed method in a representative indoor scene, an office corridor with several sunk doors. In this test scene, there are several sunk doors on both sides of the corridor and a turning corner in the end of the corridor, as shown in Figure 5(a). The traditional contour-slope model can't generate a very reasonable likelihood map representing this scene accurately mainly because that there possibly exist non-occupied regions, e.g., suck doors and the turning corner, inside the connected contours as whole walls. Therefore, in Figure 5(b), due to the errors of the contours, we found that the grids in the regions of the sunk doors and the turning corner are also connected in the occupancy likelihood map generated by the traditional contour-slope model, especially in the turning corner marked by the red number 1 . However, in the occupancy likelihood generated by our proposed method, this problem is alleviated greatly with the same connection results of contours, as shown in Figure 5(c). This comparative experiment proves that our proposed method is more reasonable than the traditional contour-slop model due to much less connection errors in contours.

To evaluate the performance of our integrated scan distortion compensation strategy, we collected a group of laser scans in a narrow and closed-loop indoor office corridor with a length of about $100 \mathrm{~m}$. Figure 6(a) shows the finally learned occupancy likelihood map and the moving trajectory generated by considering the scan distortion compensation while the results without considering it are illustrated in Figure 6(b). From Figure 6, we obviously observed that there an obvious improvement with a much less loop closure error if the scan distortion compensation was applied. Although an improvement was obtained in this experiment, there still exists a small loop closure error mainly because this test scene is so narrow and clean that the laser scanner cannot see enough features, especially near the turning corner. It can be further improved by introducing a loopclosure detection. With the increasing of moving velocity of the mobile platform, the scan distortion becomes more severe and its 


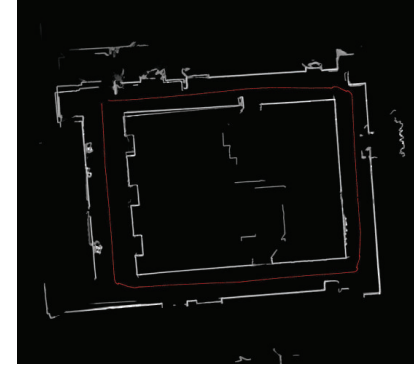

(a)

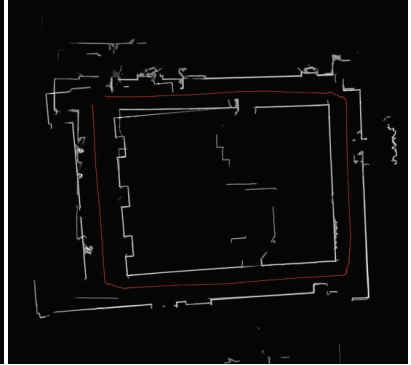

(b)
Figure 6: The occupancy likelihood maps of a narrow and closed-loop indoor office corridor with a length of about $100 \mathrm{~m}$ associated with the moving trajectories (marked in red lines) of the UGV generated by considering the scan distortion compensation in (a) and without it in (b).

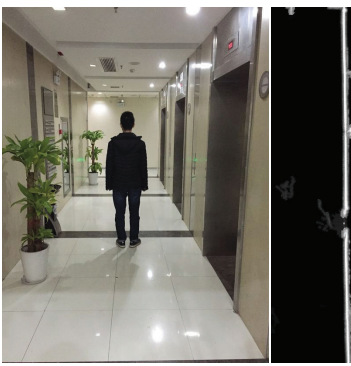

(a)

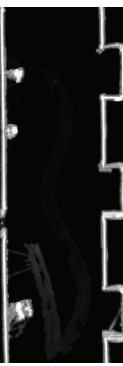

(b)

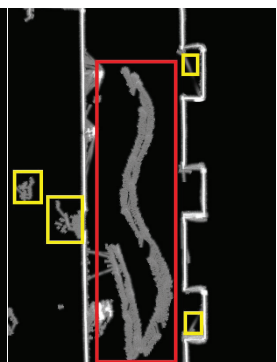

(c)
Figure 7: Comparative results of different likelihood updating strategies in an indoor office corridor with a walking pedestrian: (a) a scene photo; (b)-(c) the last likelihood maps with our proposed learning strategy with decreasing and without it, respectively. Brighter intensities indicate higher likelihood values.

compensation is very necessary.

To illustrate that our algorithm can deal with the dynamic scene more efficiently, we first tested our algorithm in an indoor office corridor, as shown in Figure 7(a). The main objects in this scene are comprised of a walking pedestrian, three elevators, two plants and the corridor walls. With our proposed occupancy likelihood map learning strategy, only static structures of this scene are visible in the finally learned occupancy likelihood map while the dynamic objects almost disappear with extremely low likelihood values, as shown in Figure 7(b). If we don't consider the deceasing strategy in the occupancy likelihood map learning process, the dynamic objects are also obviously visible in the finally learned map, as shown in Figure 7(c). Except that the main dynamic object, a walking pedestrian marked in the red box shown in Figure 7(c), disappeared in Figure 7(b), other disturbed grids marked in the yellow boxes in Figure 7(c) caused by laser data noise also disappeared in Figure 7(b). Furthermore, we tested our learning strategy in another indoor scene with multiple dynamic objects, walking pedestrians, as shown in Figure 8, from which the same conclusions can be drawn. These two comparative experiments sufficiently demonstrate that our proposed occupancy likelihood map learning strategy is much robust to dynamic objects and data noises. With such much better occupancy likelihood map, the localization would be improved to some extent.

Finally, we tested our SLAM algorithm in another narrow long indoor corridor with a length of about $100 \mathrm{~m}$. The last occupancy likelihood map and the complete moving trajectory of the mobile UGV platform generated by our proposed SLAM algorithm are illustrated in Figure 9. The mobile UGV for data acquisition

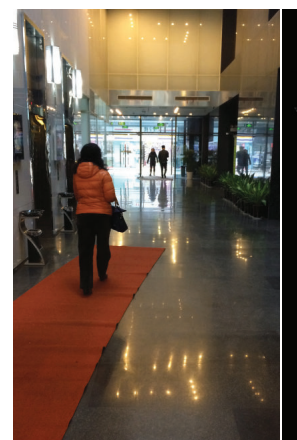

(a)

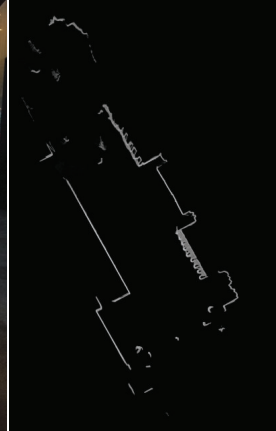

(b)

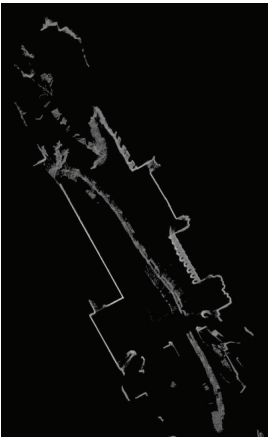

(c)
Figure 8: Comparative results of different likelihood updating strategies in an office lobby with multiple walking pedestrians: (a) a scene photo; (b)-(c) the last likelihood maps with our proposed learning strategy with decreasing and without it, respectively. Brighter intensities indicate higher likelihood values.

started at one end of the corridor, moved to another end along the corridor, and finally come back near the starting location. From the moving trajectory shown in Figure 9, we observed that there no exists the drifting error in our SLAM results for this challenged scene.

\section{CONCLUSION}

Based on the data collected by a 2D laser scanner mounted on a UGV platform, a new indoor SLAM algorithm via the laser scan matching aided by the grid-based occupancy likelihood map was proposed in this paper. Instead of computing the occupancy likelihood value of each grid based on the point-topoint distance or point-to-contour one, we combined those two measurements by applying a 2-D Gaussian blurring operation along the contours. In the scan-to-map matching process, to reduce the influence of scan distortion caused by the motion of the laser scanner, we estimated its velocity, and all points can be transformed by compensating the distortion via the estimated velocity as if they were measured at the same time, all of these ideas are integrated into the LM optimization framework to find the optimal rigid-body transformation. Besides, to ensure the accuracy and robustness of the laser scan matching, we estimated a rough initial transformation via brute search for the LM optimization and implemented the whole optimization framework in multiple resolutions. At last, inspired by background subtraction, we creatively proposed a novel strategy to update the occupancy likelihood map to greatly reduce the affect of dynamic objects often existed in most indoor scenes and data noises caused by the laser scanner itself. Experimental results on several data sets acquired from typical indoor environments demonstrate that our proposed SLAM algorithm can provide high-precision positioning and mapping results in most cases.

\section{ACKNOWLEDGMENT}

This work was supported by the National Natural Science Foundation of China (Project No. 41571436), the Hubei Province Science and Technology Support Program, China (Project No. 2015BAA027), the Jiangsu Province Science and Technology Support Program, China (Project No. BE2014866), and the South Wisdom Valley Innovative Research Team Program. 


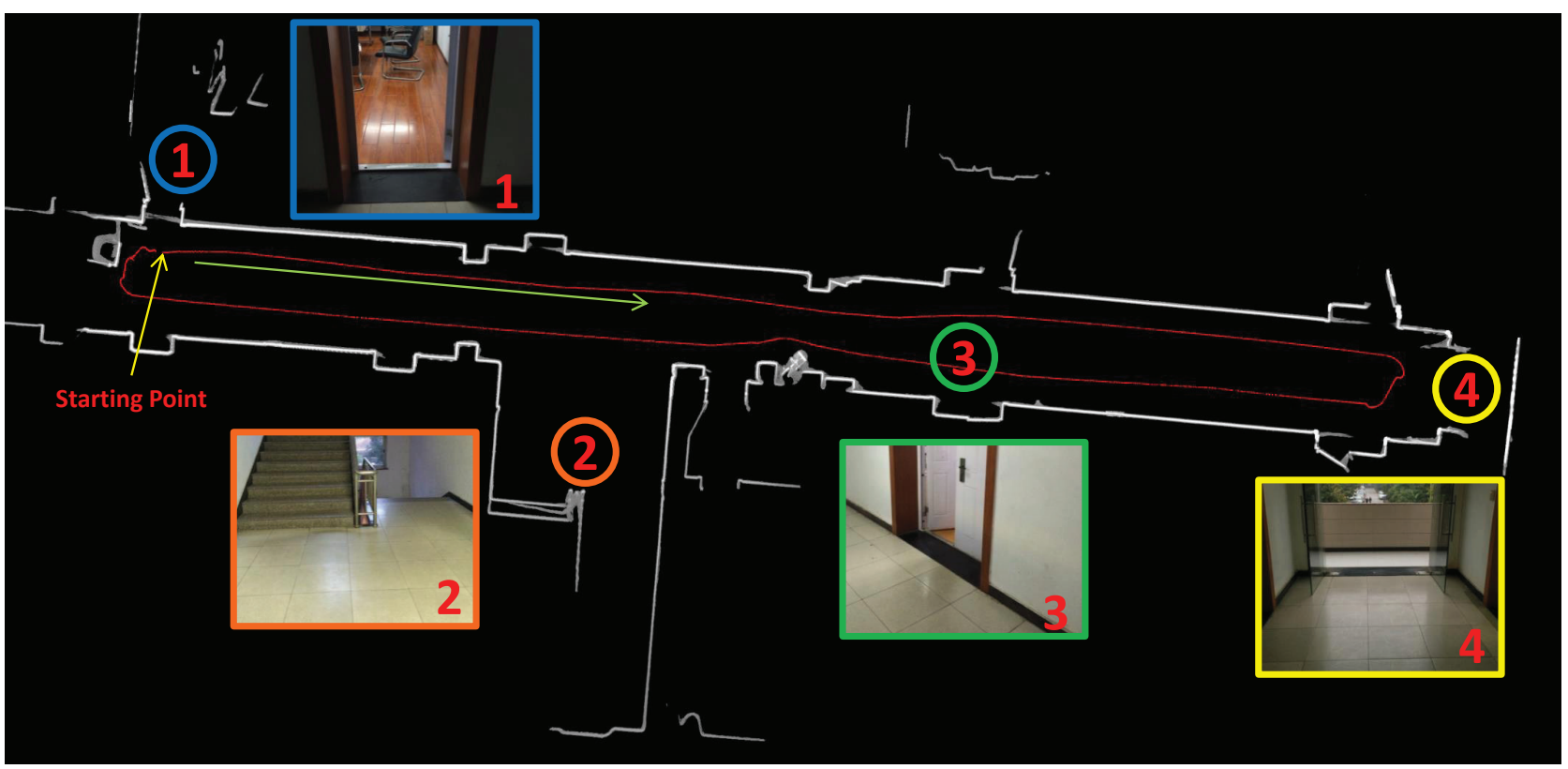

Figure 9: The occupancy likelihood map with a recovered moving trajectory (marked in red line) generated by our proposed SLAM algorithm in a narrow and long corridor with a length of about $100 \mathrm{~m}$.

\section{REFERENCES}

Bachrach, A., He, R. and Roy, N., 2009. Autonomous flight in unknown indoor environments. International Journal of Micro Air Vehicles 1(4), pp. 217-228.

Bachrach, A., Prentice, S., He, R. and Roy, N., 2011. RANGE-robust autonomous navigation in GPS-denied environments. Journal of Field Robotics 28(5), pp. 644-666.

Besl, P. J. and McKay, N. D., 1992. Method for registration of 3-D shapes. In: Robotics-DL tentative.

Bezet, O. and Cherfaoui, V., 2006. Time error correction for laser range scanner data. In: International Conference on Information Fusion.

Biber, P. and Straßer, W., 2003. The normal distributions transform: A new approach to laser scan matching. In: IEEE/RSJ International Conference on Intelligent Robots and Systems (IROS).

Bourmaud, G. and Mégret, R., 2015. Robust large scale monocular visual SLAM. In: IEEE Conference on Computer Vision and Pattern Recognition $(C V P R)$.

Censi, A., 2008. An ICP variant using a point-to-line metric. In: IEEE International Conference on Robotics and Automation (ICRA).

Choi, S., Zhou, Q.-Y. and Koltun, V., 2015. Robust reconstruction of indoor scenes. In: IEEE Conference on Computer Vision and Pattern Recognition (CVPR).

Diosi, A. and Kleeman, L., 2005. Laser scan matching in polar coordinates with application to SLAM. In: IEEE/RSJ International Conference on Intelligent Robots and Systems(IROS).

Endres, F., Hess, J., Sturm, J., Cremers, D. and Burgard, W., 2014. 3D mapping with an RGB-D camera. IEEE Transactions on Robotics 30(1), pp. 177-187.

Engel, J., Schöps, T. and Cremers, D., 2014. LSD-SLAM: Large-scale direct monocular SLAM. In: European Conference on Computer Vision $(E C C V)$, Springer.

Grisetti, G., Stachniss, C. and Burgard, W., 2007. Improved techniques for grid mapping with rao-blackwellized particle filters. IEEE Transactions on Robotics 23(1), pp. 34-46.

Hartley, R. and Zisserman, A., 2003. Multiple view geometry in computer vision. Cambridge university press.

Hong, S., Ko, H. and Kim, J., 2010. VICP: Velocity updating iterative closest point algorithm. In: IEEE International Conference on Robotics and Automation (ICRA).
Kohlbrecher, S., Von Stryk, O., Meyer, J. and Klingauf, U., 2011. A flexible and scalable SLAM system with full 3D motion estimation. In: IEEE International Symposium on Safety, Security, and Rescue Robotics (SSRR).

Lemaire, T., Berger, C., Jung, I.-K. and Lacroix, S., 2007. Vision-based SLAM: Stereo and monocular approaches. International Journal of Computer Vision 74(3), pp. 343-364

Nurunnabi, A., West, G. and Belton, D., 2015. Outlier detection and robust normal-curvature estimation in mobile laser scanning $3 \mathrm{D}$ point cloud data. Pattern Recognition 48(4), pp. 1404-1419.

Oh, T., Lee, D., Kim, H. and Myung, H., 2015. Graph structure-based simultaneous localization and mapping using a hybrid method of $2 \mathrm{D}$ laser scan and monocular camera image in environments with laser scan ambiguity. Sensors 15(7), pp. 15830-15852.

Olson, E. B., 2009. Real-time correlative scan matching. In: IEEE International Conference on Robotics and Automation (ICRA).

Olson, E. B., Teller, S. and Leonard, J., 2008. Robust and efficient robotic mapping. PhD thesis, Massachusetts Institute of Technology, Department of Electrical Engineering and Computer Science.

Rapp, M., Barjenbruch, M., Hahn, M., Dickmann, J. and Dietmayer, K., 2015. Clustering improved grid map registration using the norma distribution transform. In: IEEE Intelligent Vehicles Symposium (IV).

Steux, B. and Hamzaoui, O. E., 2010. tinySLAM: A SLAM algorithm in less than 200 lines c-language program. In: International Conference on Control Automation Robotics \& Vision (ICARCV).

Tang, J., Chen, Y., Jaakkola, A., Liu, J., Hyyppä, J. and Hyyppä, H., 2014. NAVIS-an UGV indoor positioning system using laser scan matching for large-area real-time applications. Sensors 14(7), pp. 11805-11824.

Tang, J., Chen, Y., Niu, X., Wang, L., Chen, L., Liu, J., Shi, C. and Hyyppä, J., 2015. LiDAR scan matching aided inertial navigation system in GNSS-denied environments. Sensors 15(7), pp. 16710 16728.

Yao, J. and Odobez, J.-M., 2007. Multi-layer background subtraction based on color and texture. In: IEEE Conference on Computer Vision and Pattern Recognition (CVPR).

Zhang, J. and Singh, S., 2014. LOAM: LiDAR odometry and mapping in real-time. In: Robotics: Science and Systems Conference (RSS).

Zlot, R. and Bosse, M., 2014. Efficient large-scale three-dimensional mobile mapping for underground mines. Journal of Field Robotics 31(5), pp. 758-779. 